\title{
15 IL-27 RECEPTOR SIGNALING IS CRITICAL FOR B CELL DIFFERENTIATION IN COLLAGEN INDUCED ARTHRITIS
}

Odilia B J Corneth, ${ }^{1,2}$ Anne-Marie C Mus, ${ }^{1,2}$ Patrick S Asmawidjaja, ${ }^{1,2}$ Rudi W Hendriks, ${ }^{3}$ Erik Lubberts, ${ }^{1}{ }^{1}$ Department of Rheumatology, Erasmus Medical Center, Rotterdam, The Netherlands, ${ }^{2}$ Department of Immunology, Erasmus Medical Center, Rotterdam, The Netherlands; ${ }^{3}$ Department of Pulmonary Medicine, Erasmus Medical Center, Rotterdam, The Netherlands

10.1136/annrheumdis-2011-201239.15

Background IL-27 receptor (IL-27R) signaling induces Th1 differentiation and IL-10 production and inhibits Th17 differentiation. In experimental autoimmune encephalomyelitis (EAE), IL-27R knock-out mice showed higher proliferation of draining lymph nodes with more production of IL-17, IL-6 and tumour necrosis factor $\alpha$. The authors therefore hypothesised that collagen induced arthritis (CIA) would be enhanced in IL-27R knock-out mice compared to controls.

Objective To investigate the role of IL-27R signaling in CIA. Methods IL-27R knock-out mice and controls were immunised with chicken collagen type II and complete Freunds adjuvant (CFA), and boosted again 21 days later. Mice were euthanised 10, 36 and 45 days after immunisation and B and $\mathrm{T}$ cell subsets in spleen, lymph nodes and joints were analysed using flow cytometry. Furthermore, auto-antibodies were determined in serum using ELISA and immunohistochemic analysis of the spleen was performed. For antigen induced arthritis, mice were immunised with methylated bovine serum albumin (mBSA) in CFA and a local injection of mBSA in the knee joint was given 1 week later.

Results Interestingly, both the incidence of CIA and the arthritis score were significantly lower in IL-27R knock-out mice compared to controls. In addition, synovial inflammation was markedly decreased in IL-27R knock-out mice. Although the proportion of Th17 cells was increased in IL-27R knock-out mice, the total number of CD4+T cells was significantly lower. To assess whether Th17 cells were pathogenic the authors made use of the Th17 mediated antigen induced arthritis (AIA) model. Inflammation of the knee joints in IL-27R knock-out mice in AIA was comparable to controls, showing that the pathogenicity of these cells is normal. As B cells also play a significant role in CIA, the authors then analysed $B$ cells by flow cytometry. The total number of B cells was lower in IL-27R knock-out mice. Also, IL-27R knock-out mice hardly develop germinal center $B$ cells, which the authors confirmed by immunohistochemistry. In line with this, the authors found that collagen specific IgG antibody levels were 
lower in these mice suggesting a critical role for IL-27R signaling in further differentiation of mature B cells.

Conclusion Our data show a critical role of the IL-27R signaling in the development of CIA. The development of germinal center B cells was significantly impaired. These data suggest that IL-27R signaling is involved in B cell differentiation and function and that the lack of germinal center $B$ cells in the IL-27R deficient mice is most critical for the reduced expression of CIA. 